\title{
Designing for the Secondary User Experience
}

\author{
Ole Andreas Alsos and Dag Svanæs \\ Department of Computer and Information Science, \\ Norwegian University of Science and Technology, Trondheim, Norway \\ \{Ole.Andreas.Alsos, Dag.Svanes\} @idi.ntnu.no
}

\begin{abstract}
Computer systems are first and foremost designed for primary users. The needs of the other types of users, such as secondary users, are often overlooked. These users are not interacting with the system directly but are yet affected by it. This study is based on empirical findings from two usability evaluations in a realistic hospital setting with physicians and patient actors. We have found that also secondary users, such as patients, have a kind of user experience during the primary user's interaction with the system. We conclude from this that designers and developers should also address the need of secondary users and include them in the design and evaluation process. This means designing devices or GUIs that (1) support non-verbal communication, (2) provide feedback to the secondary users, (3) use their language and representation, and (4) is tailored for the secondary user. Sometimes a focus on the secondary user implies that the designer must deal with conflicting needs between the primary and the secondary users.
\end{abstract}

Keywords: User experience, UX, end user, secondary user, secondary user experience, patient experience, clinical simulation, usability evaluation.

\section{Introduction}

Most user interfaces are designed for single users. This is true even for the user interfaces of collaborative applications, such as Facebook and Skype (although the applications are collaborative). However, as systems are being used in collaborative settings, the use of computing systems will increasingly involve people beside the primary user. Sometimes other people indirectly become users of the user interface without even interacting with it. One such situation is face-to-face interactions where one person, the primary user, is using an information system while interacting with another person face-to-face, i.e. the secondary user. It could be a physician talking with a patient in a medical consultation while interacting with an electronic patient record system, or a clerk serving a customer while looking up information in a product directory (e.g. in banks, travel agencies, or shops). In such situations one could expect that both persons are affected directly or indirectly by the user interface of the information system; both the primary user, who is using the system directly, and the secondary user, who relies on the primary user to obtain information from the system and is affected by the primary user's experiences with the system. 
The needs of the secondary users are rarely in the minds of the system designers and developers of information systems, and are often neglected [1]. While the user experience (UX) is considered important when designing for the primary user, there is no equivalent concept in the HCI literature for the secondary users.

In this paper we seek to extend focus on user experience to go beyond the traditional "first person user" perspective and to include secondary users. We do this by defining the secondary user experience. Then, by drawing on two different usability evaluation studies of mobile devices used in a realistic hospital setting, we identify some ways in which the design of the user interface can affect the secondary user experience. Finally, we provide preliminary guidelines for designing for the secondary user experience in a hospital setting.

\section{Secondary User Experience}

Eason [2] identifies three categories of users; (1) primary users who are frequent hands-on users of the system; (2) secondary users who are occasional users or use the system through an intermediary, and (3) tertiary users, who are affected by the introduction of the system or influence its purchase. Other researchers have defined other comparable end-user variations $[3,4]$.

It is the authors' impression that designers and developers to some extent are aware of the peripheral end-user groups, i.e. those who do not directly interact with the systems, but that few actually design for them. They mainly take the direct/primary user into account when designing system. Thorough work is often done in identifying stakeholders in the requirements process, but end-users are mainly considered those who directly interact with the system (e.g. [1]).

The current usability definition, ISO 9241-11:1998 [5], does not distinguish between different end-users. However, a number of HCI publications refer to a 1997 draft of the definition. This version contained a reference to people that do not directly interact with the system: "[Satisfaction is] the comfort and acceptability of the work system to its users and other people affected by its use" (our emphasis). However, this reference to "other people" was omitted in the final version of the usability standard (see [6] for an example).

In many use situations, especially from client-service relations and health care, the primary users of information systems are engaged in face-to-face interaction with customers or patients. They may for example be a client making deposits in a bank, a globetrotter booking a flight in a travel agency, or a customer buying products in a shop. The way we see it, these types of secondary users are recognized by the following characteristics:

- They are interacting with the primary user, who interacts with the system.

- They are not (or in little extent) interacting directly with the system themselves.

- They rely on the primary user to obtain information from the system.

- They are influenced by the primary user's experiences with the system (e.g. effectiveness, efficiency, satisfaction, etc.) 


\subsection{User Experience and Co-experience}

Researchers and practitioners have different views on what the user experience (UX) is [7]. As defined in ISO 9421-210 [8], it is "a person's perceptions and responses that result from the use or anticipated use of a product, system or service". UX is here associated with the primary user.

Others have proposed comparable definitions, such as Law et al., [7], who propose that "UX focuses on interaction between a person and something that has a user interface". The study excludes face-to-face interaction between people, unless a user interface is involved in the interaction. For this kind of interactions, Co-Experience provides a better explanation; it is the user experience created in social interaction with the presence of a system or product [9]. For use situations as described in the introduction, UX and co-experience relates as follows: While UX concerns the primary user, co-experience relates to both the primary and secondary user (Figure 1).

\subsection{Secondary User Experience}

We have found few examples of secondary user experiences in literature. An exception is provided by Montague [10], who claims that patients have a user experience of technology used by care providers even if they are passive users of it. During childbirth, the health care providers use technology to monitor the health condition of the unborn child. By interviewing mothers about the technology used on them, Montague found that when technology worked well, it created positive experiences and increased the patients' connection with their babies. When technology did not work well, or when care providers could not get technologies to work properly, negative experiences occurred.

We have found no existing definitions of the user experience of secondary users. Therefore we define it as follows:

The secondary user experience of a system is the part of the overall experience of the secondary user that can be attributed to (1) the primary user's interaction with the system, or (2) the secondary user's interaction with the system with the primary user as an intermediary.

Secondary UX is different from UX and co-experience in that it only relates to the experiences of the secondary user (see Figure 1).

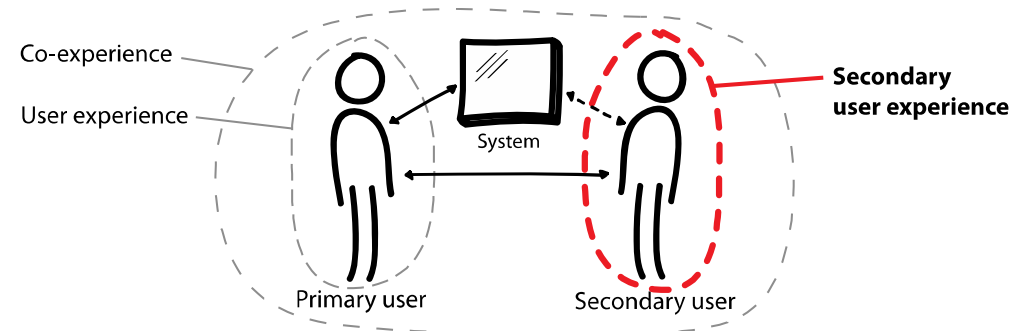

Fig. 1. $U X$ relates to the primary user, Co-experience relates to the shared experience by the system of both users, and secondary $U X$ relates to the secondary user. The solid arrows indicate interaction, while the dotted arrow indicates indirect interaction. 


\section{Methods}

The ward round is a situation with both a primary user (the doctor) and a secondary user of a system (the patient). Mobile electronic patient record systems for bedside usage are primarily designed with the doctor in mind. Few have considered the effects on the patient. Therefore, we have retrospectively analyzed the data and findings from two previously conducted usability evaluation studies of prototype mobile systems for hospitals [11, 12]. Both evaluations were conducted in a simulated hospital environment with multiple persons involved; real physicians consulting patient actors lying in the hospital beds.

Using handhelds and patient terminals together. In the first evaluation, we explored several ways of allowing doctors to use handheld devices together with bedside mounted patient terminals for viewing $\mathrm{x}$-ray images in collaboration with the patient (Figure 2). Five pairs consisting of a physician and a patient each performed eight ward rounds using different variants of the x-ray image viewer. In total, 40 ward rounds where performed. For each evaluation, the physician and the patient were interviewed simultaneously about aspects concerning the user experience of the system. The prototypes and research approach are previously described in [11].

Handheld medication system. In the second study we explored interaction techniques for a handheld medication system; one paper based and three mobile patient record systems (Figure 3). 14 physicians conducting in total 56 ward rounds. Physicians and the patients were interviewed separately about aspects concerning the user experience. The study and prototypes are previously described in [12].

With assistance from a physician and a nurse, much effort was used designing the patient scenarios, tasks and environment for both evaluations as close to their real working environment as possible. The patient actors were carefully instructed how to behave and what to say, to make the behavior as realistic as possible. Senior sociologists with experience from health care studies, as well as facilitators and participating physicians and patients considered the scenarios played out in the evaluations to be realistic instances of hospital work.

The video recordings from the evaluations were analyzed using methods from video-based studies of human interaction with technology [13], while the interview data was analyzed qualitatively using methods influenced by grounded theory.
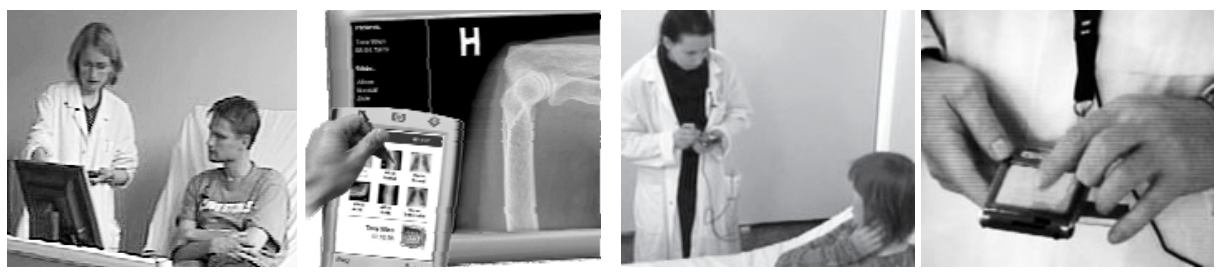

Fig. 2. Using handheld devices together with Fig. 3. Comparing interaction techniques for bedside mounted patient terminals handheld medication system 


\section{Results}

When replacing the non-digital solutions, such as paper based records and x-rays images with mobile computing technology, we found a number of issues related to the user experience for the primary and secondary users:

The primary users preferred digital over non-digital. Although the physicians in general were confident in using the paper chart (study 2), they preferred using the mobile computing device. A number of functions and attributes, such as pocket size, error prevention and undo mechanisms, contributed positively to the UX.

Secondary users had strong opinions about unused system. In the first study the patients, as passive users of the system, had strong opinions about the different versions of the $\mathrm{x}$-ray viewer, even if the patient's part of the system was identical (or nearly identical) for all versions. The main difference was on the mobile device, which was only used by the physician. However, the physicians' usage of the different variants of the system had large effects, both on how smoothly they were able to present new x-ray images on the patient terminal, and how both the physicians and patients rated them afterwards.

Mobile devices hampered the nonverbal communication. The physical form factor of the paper chart allowed the physician to use it as a channel for nonverbal communication. For example, some doctors signaled that the consultation was ending by closing the chart, or invited the patients to speak by tilting the chart towards them. This was harder with the PDA, and was considered negative by the patients.

Mobile devices reduced the doctor-patient dialogue. The user interface of the mobile device increased legibility and allowed the physicians to undo and minimize medication errors. On the other hand, the user interface gave poor information overview and had unfamiliar interaction techniques. This required much of the physicians' attention, and according to the patients it hampered the doctor-patient dialogue and reduced their satisfaction of the consultation.

Good for the doctors was (sometimes) bad for the patients. In most of the design solutions of the first study, the doctor controlled the patient terminal through a mobile device. While this was seen as a major benefit from the perspective of the physicians, who could hide private information on the mobile device and display public information on the patient terminal, it was perceived as negative from the perspective of the patients. They did not understand what was going on and did not like that information was hidden for them.

Good for the patients was (sometimes) bad for the doctors. In other design solutions the physicians controlled the system directly through the patient terminal. Unintentionally it also allowed the patient to partly interact with the terminal. For the patient this was perceived as an improvement. For the physicians, however, it became harder to control the system as they had to bend over the patient to use it. 
User interface complexity confused patients. In some versions of the first study, the controls for changing information content were present on the patient terminal. The increased complexity of the GUI confused some patients. They rather preferred the versions where these controls were moved onto the physician's mobile device.

Spoken language was influenced by GUI. In the second study, a pause symbol (as used in music and video players) was used on the mobile device to temporarily cease medical treatment without removing it from the medication list. This lead the physicians to use words like "pausing this drug" rather than the more incomprehensible term "cessation", which was commonly used when using the paper chart. While the first term was obvious for the patients, the latter was a foreign word.

\section{Discussion}

The findings in this study demonstrate that secondary users have a kind of user experience of an information system that is used by primary users. Further it shows that designers sometimes face tradeoffs between the primary and secondary UX.

\subsection{User Experience is Relevant for Secondary Users}

The studies demonstrated, not surprisingly, that technology had an impact on the primary user (i.e. physician), who was directly interacting with the system. Further, our observations showed that the primary users' interaction with the system also had an impact on the secondary users. For example, the patients had some sort of user experience; they had strong perceptions and responses about the system, although they had not used the systems directly themselves.

The positive correlation between patient satisfaction on health outcome has long been established [14]. When patients report that they are satisfied or dissatisfied because of the physician's interaction with the system (i.e. their experiences as a secondary user), we can assume that it has some impact on the overall patient satisfaction. This was for example seen when the complexity of the GUI confused patients.

\subsection{Trade-Off between Primary and Secondary User Experience}

The analysis indicates that designers are faced with tradeoffs between the needs of the primary and secondary users. When the user experience was improved for the physicians, it had in some cases negative effects for the patients, e.g. the ability to hide information on the mobile device. In addition, we found that when the secondary user experience was improved, it sometimes created new problems for the physicians, e.g. reducing the ergonomics when interacting with the system. Consequently, aspects of the user experience for the primary user can have negative consequences for the secondary user. In a similar manner, improving the user experience for the secondary user can have negative consequences for the primary user.

How should one deal with the potential trade-offs between the design of the primary and secondary user experience? One cannot design the primary user experience first and then the secondary user experience. It may lead to a suboptimal 
solution for the latter. Both user experiences must therefore be designed together. Yet, the designers must often prioritize. In the hospital it could be a bad priority to propose designs that threat patient safety just to make the patient experience slightly better.

\subsection{First Steps towards Design Guidelines}

Accommodating the needs of the secondary users is important. In the context of a ward round with a physician and patients, a positive secondary user experience can have positive effect on the doctor patient dialogue, which is important for the treatment and care of the patients [14]. In the context of business, for example a travel agent serving a potential traveler, or a checker handling a customer, improving the secondary user experience can have positive effect on the overall customer experience. This often means satisfied returning customers and increased revenue [15]. Drawing on our findings, we suggest four preliminary design guidelines that we find relevant for information systems involving a secondary user experience. Research in other domains and with other technologies will be needed to make the list more complete:

- Give system feedback to the secondary user. By increasing the action transparency (i.e. increase the visibility of actions) or providing alternative system feedback to the secondary user, one can improve the secondary user experience.

- Support non-verbal communication. The quality of the non-verbal aspects of faceto-face communication has a strong impact on the secondary user experience. Our findings indicate that the system can hinder this communication, especially when the system occupies the hands or hides the face of the primary user. Therefore, the physical form factor of the system needs to support nonverbal communication.

- Use the language and representation of the secondary user. By presenting the information for the primary user in the language of the secondary user, the primary user can be guided to use simpler terms and communicate on the same level as them, i.e. physicians use terms like "blood sugar level" instead of "glucose". This can make it easier for the secondary user to understand.

- Provide a GUI and/or device tailored for the secondary user. If feasible and necessary, an additional device/GUI with information tailored for the secondary user should be provided. This will give the secondary users a version of the information where unnecessary complexity and irrelevant information is removed.

In addition to the above suggestions, we suggest that (1) the perspectives of the secondary user are included throughout the design process, and that (2) usability evaluations include both the primary and secondary users together.

\subsection{Limitations and Future Work}

It is important to note that the evaluation studies presented here were not explicitly designed for the purpose of illuminating the secondary user experience. The data was analyzed retrospectively. Moreover, we recognize the limitations regarding the use of "role-plays" as one of our primary data sources.

The design guidelines are very preliminary and their validity is limited to ward round scenarios with mobile technology. We do believe, however, that other domains with secondary users may benefit from similar studies to improve the secondary user 
experience. Future work should further investigate the concept of secondary user experience, as well as the generalizability outside the presented case.

\section{Conclusion}

The needs of secondary users, who only use an information system occasionally or through an intermediary, are often neglected. Designers and developers must address the need of secondary users and include them in the design and evaluation process of the system. This means designing devices or GUIs that (1) support non-verbal communication, (2) provide feedback to the secondary users, (3) use their language and representations, and (4) is tailored for the secondary user. Sometimes this implies that the designers deal with conflicting needs between primary and secondary users.

\section{References}

1. Sharp, H., Finkelstein, A., Galal, G.: Stakeholder identification in the requirements engineering process. In: Proceedings DEXA 1999. IEEE, Los Alamitos (1999)

2. Eason, K.: Information Technology and Organisational Change. Taylor and Francis, Abington (1987)

3. Faulkner, X.: Usability Engineering. Palgrave Macmillan, Basingstoke (2000)

4. Ågerfalk, P.J.: Who is the user in user-centered design? In: HCII. Springer, Heidelberg (2001)

5. ISO 9241-11:1998. Ergonomics of human system interaction - Part 11: Guidance on usability specification and measures. International Organization for Standardization

6. O’Grady, M.J., O'Hare, G.M.P., Sas, C.: Mobile agents for mobile tourists: a user evaluation of Gulliver's Genie. Interacting with Computers 17 (2005)

7. Law, E.L.-C., Roto, V., Hassenzahl, M., et al.: Understanding, scoping and defining user experience: a survey approach. In: Proceedings of CHI 2009. ACM, New York (2009)

8. ISO 9241-210:2009. Ergonomics of human system interaction - Part 210: Human-centred design for interactive systems. International Organization for Standardization

9. Battarbee, K.: Defining Co-Experience. In: Proceedings of DPPI 2003. ACM, New York (2003)

10. Montague, E.N.H.: Understanding Patient User Experience in Obstetric Work Systems. Health (San Francisco), 70-77 (2009)

11. Alsos, O.A., Svanæs, D.: Interaction techniques for using handhelds and PCs together in a clinical setting. In: Proceedings of NordiCHI 2006, pp. 125-134. ACM, New York (2006)

12. Alsos, O.A., Dabelow, B., Faxvaag, A.: Doctors' Concerns of PDAs in the Ward Round Situation - Lessons from a Formative Simulation Study. Methods Inf. Med. 48(6) (2009)

13. Heath, C., Hindmarsh, J., Luff, P.: Video in qualitative research. SAGE Pub. Inc., Thousand Oaks (2010)

14. Ong, L.M.L., de Haes, J.C.J.M., Hoos, A.M., et al.: Doctor-patient communication: A review of the literature. Social Science \& Medicine 40(7), 903-918 (1995)

15. Peppers, D., Rogers, M.: Return on customer: creating maximum value from your scarcest resource. Currency (2005) 